\title{
Glueball Mass Spectrum and Mass Splitting in $2+1$ Strongly Coupled Lattice Gauge Theories
}

Michael O'Carroll, Gastão de Almeida Braga, and Ricardo S. Schor

Departmento de Física-ICEx, Universidade Federal de Minas Gerais, Belo Horizonte, Minas Gerais, Brasil

Abstract. For a $2+1$ strongly coupled $\left(\beta=2 / g^{2}\right.$ small) Wilson action lattice gauge theory with complex character we analyze the mass spectrum of the associated quantum field theory restricted to the subspace generated by the plaquette function and its complex conjugate. It is shown that there is at least one but not more than two isolated masses and each mass admits a representation of the form $m(\beta)=-4 \ln \beta+r(\beta)$, where $r(\beta)$ is a gauge group representation dependent function analytic in $\beta^{1 / 2}$ or $\beta$ at $\beta=0$. For the gauge group SU(3) there is mass splitting and the two masses $m_{ \pm}$are given by

$$
m_{ \pm}(\beta)=-4 \ln \beta+\ln 16 r^{4}+\frac{1}{2}(2 \pm 1) \beta+\left(d_{ \pm}(\beta) \equiv \sum_{n=2}^{\infty} c_{n}^{ \pm} \beta^{n}\right),
$$

where $r=3$ is the dimension of the representation and $d_{ \pm}(\beta)$ is analytic at $\beta=0$. $c_{n}^{ \pm}$can be determined from a finite number of the $\beta=0$ Taylor series coefficients of finite lattice truncated plaquette-plaquette correlation function at a finite number of points.

\section{Introduction}

In [1] the low lying energy-momentum spectrum of the quantum field theory associated with the $2+1$ strongly coupled lattice gauge theory with Wilson action $A^{\prime}$ is analyzed. Formally $A^{\prime}=\beta \sum_{p} \chi\left(g_{p}\right)$, where $\chi$ is the real character of an irreducible representation of a compact gauge group. $g_{p}$ is the oriented product of group elements around the border of the plaquette $P$. It is shown that for $\beta>0$ and small the energy-momentum spectrum in the gauge invariant subspace generated by the time zero plaquette functions $\chi\left(g_{p_{x}}\right), x=\left(x_{1}=0, \mathbf{x}\right) \in Z^{3}$ consists of an isolated dispersion curve $\omega(\mathbf{p}) \geqq \omega(\mathbf{0})$, real analytic in $\mathbf{p} \in(-\pi, \pi]^{2}$, which is identified as a glueball. Furthermore the glueball dispersion curve and mass $m \equiv \omega(\mathbf{0})$ satisfy

$$
\lim _{\beta \downarrow 0} \frac{m}{-4 \ln \beta}=1, \quad \lim _{\beta \downarrow 0} \frac{\omega(\mathbf{p})}{m}=1,
$$

uniformly in $\mathbf{p} \in(-\pi, \pi]^{2}$. 
The above results follow from decay and $\beta$ analyticity properties of the truncated plaquette-plaquette correlation function (hereafter abbreviated $\mathrm{cf}$ ) and the faster $x_{1}$ (imaginary time) decay of its convolution inverse. In [2] it is shown that $A^{\prime}$ is a quasi-analytic perturbation of a non-degenerate level in the sense that $m=$ $-4 \ln \beta+r(\beta)$, where $r(\beta)$ is a group representation dependent function analytic at $\beta=0 . c_{n}$, the $n$th $\beta=0$ Taylor series coefficient of $r(\beta)$, can be computed by a finite algorithm.

Similar results for spin systems can be found in [3]. Numerical calculations of glueball masses can be found in $[4,5]$.

Here we consider the mass spectrum for the case of a complex character (also denoted by $\left.\chi=\chi_{r}+i \chi_{i}\right)$, with the formal Wilson action $\beta \sum_{p} \operatorname{Re} \chi\left(g_{p}\right)$, restricted to the subspace generated by $\chi_{r}\left(g_{p_{x}}\right)$ and $\chi_{i}\left(g_{p_{x}}\right), x=(0, \mathbf{x})$. We state our main results concerning the mass spectrum as Theorems A and B. In what follows there exists a constant $c^{\prime}>0$ such that the results hold in the mass interval $\left(0,-5 \ln c^{\prime} \beta\right)$, $c^{\prime} \beta<1$.

Theorem A. For $\beta>0$ and small there is at least one but not more than two isolated points in the mass spectrum and each mass admits a representation of the form $m(\beta)=-4 \ln \beta+r(\beta)$, where $r(\beta)$ is an analytic function of $\beta^{1 / 2}$ or $\beta$ at $\beta=0$ and dependent on the representation of the gauge group.

Remarks. 1. Here we are dealing with a quasi-analytic perturbation of an asymptotically degenerate level.

2. Similar to $[1,2]$ the theorem follows from decay and analyticity properties of a $2 \times 2$ matrix-valued truncated plaquette-plaquette of and the faster $x_{1}$ (imaginary time) decay of its matrix-valued convolution inverse.

3. For an abelian group $r(\beta)$ is analytic in $\beta$.

By a more detailed analysis we obtain information on $\beta$ analyticity (rather than just $\beta^{1 / 2}$ analyticity) and mass splitting. Define $n_{D}, n_{\bar{D}}=0,1,2, \ldots$ by

$n_{D}\left(n_{\bar{D}}\right)=$ the number of times the representation (complex conjugate representation) of the gauge group occurs in the decomposition of the Kronecker product of the representation with itself (see Lemma IV.1a and b).

We have

Theorem B. If $n+n_{\bar{D}}>0$, then there are two distinct masses, $m_{ \pm}$, given by

$$
m_{ \pm}(\beta)=-\ln \beta^{4}+\ln 16 r^{4}-\frac{1}{2}\left[(2 \pm 1) n_{\bar{D}}+(8 \pm 1) n_{D}\right] \beta+\sum_{n=2}^{\infty} c_{n}^{ \pm} \beta^{n}
$$

where $\sum_{n=2}^{\infty} c_{n}^{ \pm} \beta^{n}$ are analytic at $\beta=0$. In particular, for $\mathrm{SU}(3)$

$$
m_{ \pm}(\beta)=-\ln \beta^{4}+\ln 16 r^{4}-\frac{1}{2}(2 \pm 1) \beta+0\left(\beta^{2}\right), \quad r=3
$$

$c_{n}^{ \pm}$are group representation dependent constants that are determined from a finite number of the $\beta=0$ Taylor series coefficients of finite lattice two-point cf's at a finite number of points. 
Remark. 1. The determination of $c_{n}^{ \pm}$is a finite problem. The Taylor series coefficients of the two-point cf can be obtained from differentiating $\ln Z_{A}^{\prime}$ with respect to parameters where $Z_{A}^{\prime}$ is an appropriate partition function for a finite lattice $\Lambda$. By using the polymer expansion of [7] only a finite number (dependent on $n$ but not on $\Lambda$ ) of polymers are needed in $\ln Z_{\Lambda}^{\prime}$. In this way the troublesome problem of cancellation of $\Lambda$ dependent terms is avoided.

2. For an abelian group $n_{D}=n_{\bar{D}}=0$. However, we can show that there are masses $m_{ \pm}(\beta)$ (not necessarily distinct) with

$$
m_{ \pm}(\beta)=-4 \ln \beta+\ln 16+\left(\sum_{n=2}^{\infty} c_{n}^{ \pm} \beta_{n} \equiv d_{ \pm}(\beta)\right)
$$

and $d_{ \pm}(\beta)$ analytic. Thus if mass splitting occurs it is at least of order $\beta^{2}$.

3. The mass splitting can be interpreted as "parity" splitting since a reflection of one space component in space dimension two inverts the orientation of plaquettes perpendicular to the imaginary time direction.

We describe the organization of the paper. In Sect. II we obtain a spectral representation for the Fourier transform of the cf and give a criteria for a point to belong to the mass spectrum. In Sect. III we establish an implicit equation for the mass and prove Theorem A; in Sect. IV we prove Theorem B. Decay and $\beta$ analyticity properties of the cf and its convolution inverse used in Sect. II-IV are established in Sect. V. Section VI is devoted to some concluding remarks.

As many of the proofs are adaptations of those of $[1,2]$ we will be rather sketchy. $|\beta|$ will be assumed to be small throughout and $c, c^{\prime}, c_{1}, \ldots$ will denote strictly positive constants.

\section{Spectral Considerations}

We consider the $2 \times 2$ matrix-valued truncated plaquette-plaquette cf $G(x, \beta)$ with matrix elements

$$
G_{\alpha \gamma}(x, \beta) \equiv G_{\alpha \gamma}(y ; z, \beta)=\lim _{\Lambda \uparrow Z^{3}} G_{\Lambda \alpha \gamma}(y ; z, \beta), \quad x=y-z, x, y, z \in \Lambda \subset Z^{3},
$$

$\alpha, \gamma=r, i$. Here

$$
G_{\Lambda \alpha \gamma}(y ; z, \beta)=\left\langle\chi_{\alpha}\left(g_{p_{y}}\right) \chi_{\gamma}\left(g_{p_{z}}\right)\right\rangle_{\Lambda}-\left\langle\chi_{\alpha}\left(g_{p_{y}}\right)\right\rangle_{\Lambda}\left\langle\chi_{\gamma}\left(g_{p_{z}}\right)\right\rangle_{\Lambda},
$$

where $\chi=\chi_{r}+i \chi_{i}$ is a complex character of an $r$-dimensional irreducible unitary representation of the compact gauge group. $P_{x}$ is the translation by $x$ of the plaquette $P_{0}$ located at $x=0$ and perpendicular to the 1-direction (which is taken as imaginary time). The $\langle\cdot\rangle_{A}$ are finite lattice averages in the Gibbs ensemble with Boltzmann factor $\exp \left(\beta \sum_{p} \operatorname{Re} \chi\left(g_{p}\right)\right)$ and measure $d g_{\Lambda}$ the product of Haar measures $d \mu$ of the gauge group, one for each bond of $\Lambda$. The oriented product of $g$ 's in the Boltzmann factor and in the definition of $G_{\Lambda \alpha \gamma}$ is taken in accordance with the right-hand rule which by definition is called positive. We point out that $g_{p}^{-1}$ corresponds to the product with the opposite orientation of $g_{p}$ and that unlike the case of a real character where $\chi_{r}\left(g_{p}^{-1}\right)=\chi_{r}\left(g_{p}\right)$ a complex character satisfies $\chi_{i}\left(g_{p}^{-1}\right)=-\chi_{i}\left(g_{p}\right)$. Thus some convention is necessary in the definition of 
$G_{\Lambda \alpha \gamma}$. The existence, $\beta$ analyticity and translation invariance of the $\Lambda \uparrow Z^{3}$ limit is established in [6] (or see [7]) for small $|\beta|$. We denote points $x \in Z^{3}$ by $x=\left(x_{1}, \mathbf{x}\right), \quad \mathbf{x}=\left(x_{2}, x_{3}\right) \quad$ and $\quad|x|=\sum_{i=1}^{d}\left|x_{i}\right|=\left|x_{1}\right|+|\mathbf{x}|$. Let $\sim$ denote the Fourier transform, i.e. $\widetilde{G}(p, \beta)=\sum_{x} e^{i p x} G(x, \beta)$, where $p x=\sum_{i=1}^{3} p_{i} x_{i}, p=\left(p_{1}, \mathbf{p}\right)$. We denote by $\Gamma_{\alpha \gamma}(x, \beta) \equiv \Gamma_{\alpha \gamma}(y ; z, \beta), x=y-z$, the matrix elements of the convolution inverse, i.e. $\Gamma_{\alpha \gamma}(x ; y)=G_{\alpha \gamma}^{-1}(x ; y)$ or

$$
\sum_{z, \rho} \Gamma_{\alpha \rho}(x ; z) G_{\rho \gamma}(z ; y)=\delta_{x y} \delta_{\alpha \gamma}
$$

G, $\Gamma$ can be interpreted as matrix operators in the sequence Hilbert space $l_{2}$ with elements $f=\underset{x}{X} f_{\alpha_{x}}(x) \in l_{2}$ and norm $|f|=\left(\sum_{x, \alpha}\left|f_{\alpha_{x}}(x)\right|^{2}\right)^{1 / 2}$. We let $\|\cdot\|$ denote the $l_{2}$ operator norm.

The lattice quantum field theory Hilbert space, with inner product denoted by (,), the energy-momentum and field operators are constructed as in $[7,8]$. We denote by

$$
E\left(\lambda_{1}\right) \quad \text { and } \quad F\left(\lambda_{i}\right)=\prod_{i=2}^{3} F_{i}\left(\lambda_{i}\right), \quad \lambda=\left(\lambda_{2}, \lambda_{3}\right) \in(-\pi, \pi]^{2},
$$

the spectral resolution of the self-adjoint evolution operator (renormalized transfer "matrix") and unitary space translation operators, respectively. As in [1] we have the Feynman-Kac formula

$$
G_{\alpha \gamma}(x, \beta)=\int_{[0,1)(-\pi, \pi]^{2}} \lambda_{1}^{\left|x_{1}\right|} e^{i \lambda \cdot x} d \mu_{\alpha \gamma}\left(\lambda_{1}, \lambda\right)
$$

where

$$
\mu_{\alpha \gamma}\left(\lambda_{1}, \lambda\right)=\left(\hat{\chi}_{\alpha}\left(g_{p_{0}}\right) \Omega, E\left(\lambda_{1}\right) F(\lambda) \hat{\chi}_{\gamma}\left(g_{0}\right) \Omega\right)-\left(\hat{\chi}_{\alpha}\left(g_{p_{0}}\right) \Omega, \Omega\right)\left(\Omega, \hat{\chi}_{\gamma}\left(g_{p_{0}}\right) \Omega\right) .
$$

and $\hat{\chi}_{\alpha}\left(g_{p_{0}}\right) \Omega(\Omega)$ is the Hilbert space vector associated with $\chi\left(g_{p_{0}}\right)(1)$. $[0,1)$ can be replaced by $\left[0, e^{+4 \ln c \beta}\right)$ using the falloff of $G_{\alpha \gamma}\left(x=\left(x_{1}, 0\right)\right)$ given by Lemma 5.2. Adapting the proof of the results of [8] we have

Lemma 11.1. For each $\beta>0$ and $\mathbf{p} \in(-\pi, \pi]^{2}$ there exist signed finite measures $d \rho_{\alpha \gamma}\left(\lambda_{1}, \mathbf{p}\right)$, positive for $\alpha=\gamma$, such that

$$
\tilde{G}_{\alpha \gamma}(p)=\int_{\left[0, e^{\left.-\omega_{\alpha \gamma}(\mathbf{p})\right]}\right.}\left[\frac{1-\lambda_{1}^{2}}{1-2 \lambda_{1} \cos p_{1}+\lambda_{1}^{2}}\right] d \rho_{\alpha \gamma}\left(\lambda_{1}, \mathbf{p}\right),
$$

where

$$
\begin{aligned}
\omega_{\sigma \sigma}(\mathbf{p}) & \equiv \lim _{x_{1} \rightarrow \infty}-\frac{1}{x_{1}} \ln \left(\sum_{x} G_{\sigma \sigma}\left(x=\left(x_{1}, \mathbf{x}\right)\right) e^{i \mathbf{p} \cdot \mathbf{x}}\right) \\
& \geqq \omega_{\sigma \sigma}(\mathbf{0})=\lim _{x_{1} \rightarrow \infty}-\frac{1}{x_{1}} \ln G_{\sigma \sigma}\left(x=\left(x_{1}, \mathbf{0}\right)\right) \equiv m_{\sigma \sigma}>-4 \ln c \beta,
\end{aligned}
$$

$\omega_{\alpha \gamma}(\mathbf{p})=\min \left\{\omega_{\alpha \alpha}, \omega_{\gamma \gamma}\right\}$ for $\alpha \neq \gamma$. Furthermore for a product of intervals $\Delta_{1} \times \Delta$,

$$
\int_{\Delta_{1}} d \rho_{\alpha \alpha}\left(\lambda_{1}, \mathbf{p}\right)
$$


is continuous in $\mathbf{p} \in(-\pi, \pi]^{2}$ and

$$
\mu_{\alpha \alpha}\left(\Delta_{1} \times \Delta\right)=\int_{\Delta}\left[\int_{\Delta_{1}} d \rho_{\alpha \alpha}\left(\lambda_{1}, \mathbf{p}\right)\right] d \mathbf{p} .
$$

Remarks. 1. The importance of the above formula is that it relates the energymomentum spectrum, i.e. the support of $d \mu_{\alpha \alpha}$, to the support of the measures $d \rho_{\alpha \alpha}\left(\lambda_{1}, \mathbf{p}\right)$.

2. Formally $d \rho_{\alpha \gamma}\left(\lambda_{1}, \mathbf{p}\right)=\int_{(-\pi, \pi]^{2}} \delta(\lambda-\mathbf{p}) d \mu_{\alpha \gamma}\left(\lambda_{1}, \lambda\right)$.

From the above lemma we see that to locate the mass spectrum it is enough to determine the support of the measures $d \rho_{\alpha \alpha}\left(\lambda_{1}, \mathbf{p}=0\right)$. From now on we suppress the $\mathbf{p}$ dependence and take $\mathbf{p}=0$. We now express $\widetilde{G}_{\alpha \gamma}(p)$ in a more convenient "resolvent" form by introducing the spectral parameter $a$ and measures $d v_{\alpha \gamma}(a)$ defined by

$$
\begin{aligned}
d v_{\alpha \gamma}(a) & =\frac{1-\lambda(a)^{2}}{2 \lambda(a)} d \rho_{\alpha \gamma}(\lambda(a)), \\
a(\lambda) & =(1-\lambda)^{2} / 2 \lambda, \quad a\left(e^{-m_{\alpha \beta}}\right)=\cosh m_{\alpha \beta}-1,
\end{aligned}
$$

so that $\widetilde{G}(p)=F\left(z=\cos p_{1}-1\right)$, where we define

$$
F_{\alpha \gamma}(z)=\rho_{\alpha \gamma}(\{\lambda=0\})+\int_{\cosh m_{\alpha \gamma}-1}^{\infty} \frac{d v_{\alpha \gamma}(a)}{a-z} .
$$

$F_{\alpha \gamma}(z)$ is analytic in $z \in C-\left[\cosh m_{\alpha \gamma}-1, \infty\right)$ and we have set $m_{\alpha \gamma}=\omega_{\alpha \gamma}(\mathbf{0})$.

We recall the well-known inversion formula in

Lemma 11.2. If $c, d(c<d)$ are points of continuity of $d v_{\alpha \alpha}$, then

$$
v_{\alpha \alpha}(d)-v_{\alpha \alpha}(c)=\lim _{\varepsilon \downarrow 0} \frac{1}{i \pi} \int_{c}^{d}\left[F_{\alpha \alpha}(v+i \varepsilon)-F_{\alpha \alpha}(v-i \varepsilon)\right] d v .
$$

Remark. The representation of $F(z)$ and the inversion formula can be used in the spectral analysis of $[1,2,8,9]$ making the representation theorem for Herglotz functions unnecessary. In the non-matrix case $\operatorname{Im} F(z)^{-1}<0$ for $\operatorname{Im} z>0$ since $\operatorname{Im} F(z)>0$ for $\operatorname{Im} z>0$ from the representation for $F(z)$. Furthermore for $\operatorname{Im} z=0$, $\operatorname{Re} z$ sufficiently negative, $\operatorname{Im} F(z)=0$ so that $\operatorname{Im} F(z)^{-1}=0$ and using the CauchyRiemann equations we conclude that $F^{-1}$ is monotone. Thus in the $F^{-1}$ analyticity region $F^{-1}$ has at most one zero. $F^{-1}$ analyticity is used in the inversion formula by setting

$$
F(v+i \varepsilon)-F(v-i \varepsilon)=\frac{1}{F(v+i \varepsilon)^{-1}}-\frac{1}{F(v-i \varepsilon)^{-1}} .
$$

Let $H(z)$ be the matrix inverse of $F(z)$, i.e. $H F=I$, and $F_{\alpha \alpha}(z)=H_{\gamma \gamma}(z) / \operatorname{det} H(z)$, $\alpha \neq \gamma$. Note that $H\left(z=\cos p_{1}-1\right)=\tilde{\Gamma}\left(p_{1}, \mathbf{p}=0\right)$ and that by Lemma $5.3 \tilde{\Gamma}\left(p_{1}\right.$, $\mathbf{p}=0)$ and $\operatorname{det} \widetilde{\Gamma}\left(p_{1}, \mathbf{p}=0\right)$ are analytic in $0<\left(\operatorname{Im} p_{1}\right)<-5 \ln c^{\prime} \beta$ so that by $F_{\alpha \alpha}=H_{\gamma \gamma} / \operatorname{det} H$ and Lemma 11.2 we conclude that the mass spectrum is discrete 
in $\left(0,-5 \ln c^{\prime} \beta\right)$. We give criteria for a point $p_{1}=\mathrm{im}$ to, or not to, belong to the mass spectrum $\sigma(M)$.

Lemma 11.3. Let $0<m<-5 \ln c^{\prime} \beta$.

a) If $\operatorname{det} \tilde{\Gamma}\left(p_{1}=\mathrm{im}\right) \neq 0$, then $m \notin \sigma(M)$,

b) If $\operatorname{det} \tilde{\Gamma}\left(p_{1}=\mathrm{im}\right)=0$, and $\tilde{\Gamma}_{\alpha \alpha}\left(p_{1}=\mathrm{im}\right) \neq 0, \alpha=i$ or $r$ then $m \in \sigma(M)$.

Remarks. 1. It can't happen that $\operatorname{det} \tilde{\Gamma}\left(p_{1}\right)=0$ and $\widetilde{\Gamma}_{\alpha \alpha}\left(p_{1}\right) \neq 0, \alpha=r$ or $i$, for $0<\operatorname{Im} p_{1}<-5 \ln c^{\prime} \beta, \quad\left|\operatorname{Re} p_{1}\right|<\pi, \quad$ but $\operatorname{Re} p_{1} \neq 0 \quad$ since $\tilde{G}_{\alpha \alpha}=\widetilde{\Gamma}_{\gamma \gamma} / \operatorname{det} \tilde{\Gamma}$, $\alpha \neq \gamma$, is analytic at these points.

2 . In the case of an $n$-fold asymptotically degenerate level the spectral analysis is similar with $F_{\alpha \alpha}=H_{\alpha \alpha}^{-1}=L_{\alpha \alpha} /$ det $H$, where $L_{\alpha \alpha}$ is the cofactor matrix of $H$.

In the next section we introduce an implicit equation for the zeroes of $\operatorname{det} \tilde{\Gamma}\left(p_{1}\right)$ and prove Theorem A.

\section{Implicit Mass Equation and Proof of Theorem A}

We obtain a $\beta=0$ Taylor expansion of $\tilde{\Gamma}\left(p_{1}, \beta\right) \equiv \tilde{\Gamma}\left(p_{1}, \mathbf{p}=0, \beta\right)$ with the terms up to and including $\beta^{5}$ made explicit by first obtaining an expansion for $\widetilde{G}\left(p_{1}, \beta\right) \equiv$ $\widetilde{G}\left(p_{1}, \mathbf{p}=0, \beta\right)$. From the expansion for $\tilde{\Gamma}\left(p_{1}, \beta\right)$ we introduce an implicit equation for the mass which is used to prove Theorem A. We define

$$
G_{s}(x, \beta) \equiv G(x, \beta)-\sum_{m=0}^{5} \frac{\beta^{5}}{m !} \frac{\partial^{m} G}{\partial \beta^{m}}(x, \beta=0)
$$

$\Gamma_{s}, \widetilde{G}_{s}$ and $\tilde{\Gamma}_{s}$ are defined similarly. We have

Theorem III.1. For $\left|\operatorname{Im} p_{1}\right|<-4 \operatorname{lnc} \beta, \widetilde{G}\left(p_{1}, \beta\right)$ is jointly analytic and has the $\beta=0$ Taylor expansion

$$
\begin{aligned}
& \widetilde{G}_{\alpha \alpha}\left(p_{1}, \beta\right)=\frac{1}{2}+g_{\alpha_{1}} \beta+\cdots g_{\alpha_{5}} \beta^{5}+\left(\frac{\beta^{4}}{32 r^{4}}+\alpha_{\alpha} \beta^{5}\right)\left(e^{-i p_{1}}+e^{i p_{1}}\right)+\widetilde{G}_{s \alpha \alpha}\left(p_{1}, \beta\right), \\
& \widetilde{G}_{r i}\left(p_{1}, \beta\right)=a_{1} \beta+\cdots a_{5} \beta^{5}+\alpha \beta^{5}\left(e^{-i p_{1}}+e^{i p_{1}}\right)+\widetilde{G}_{s r i}\left(p_{1}, \beta\right) .
\end{aligned}
$$

\section{Furthermore}

$$
\begin{aligned}
\operatorname{det} \tilde{G}\left(p_{1}, \beta\right) & \equiv \widetilde{G}_{r r}\left(p_{1}, \beta\right) \widetilde{G}_{i i}\left(p_{1}, \beta\right)-\widetilde{G}_{r i}\left(p_{1}, \beta\right)^{2} \\
& =\frac{1}{4}+A_{1} \beta+\cdots A_{5} \beta^{5}+\left(\frac{\beta^{4}}{32 r^{4}}+\gamma \beta^{5}\right)\left(e^{-i p_{1}}+e^{i p_{1}}\right)+0\left(\beta^{6}\right) .
\end{aligned}
$$

Here

$$
\begin{aligned}
\gamma & =\frac{1}{2}\left(\alpha_{i}+\alpha_{r}\right)+\frac{1}{32 r^{4}}\left(g_{r_{1}}+g_{i_{1}}\right), \\
g_{r_{1}} & =\int \chi_{r}^{3} d \mu, \quad g_{i_{1}}=\int \chi_{i}^{2} \chi_{r} d \mu, \quad a_{1}=\int \chi_{i} \chi_{r}^{2} d \mu, \\
\alpha_{r} & =\frac{g_{r_{1}}}{4 r^{4}}, \quad \alpha_{i}=\frac{1}{8 r^{4}}\left(g_{i_{1}}+g_{r_{1}}\right), \quad \alpha=a_{1} / 4 r^{4} ;
\end{aligned}
$$


$g_{\alpha_{i}}, a_{i}$ are group representation constants and

$$
A_{1}=\frac{1}{2}\left(g_{i_{1}}+g_{r_{1}}\right) ; \quad A_{k}=A_{k}\left(g_{r_{k}}, \ldots, g_{r_{1}} ; g_{i_{k}}, \ldots, g_{i_{1}} ; a_{k-1} \ldots a_{1}\right), \quad 2 \leqq k \leqq 5 .
$$

Remarks. 1. To prove Theorem A only a fourth order expansion is needed, but the fifth order expansion is used in the proof of Theorem B.

2. The unexplicited constants can be made explicit as in [2] but will not be needed here.

Proof. Follows from Lemmas V.1 and V.2.

For $n=0,1,2, \ldots$ define

$$
\Gamma_{\alpha \gamma}(n, \beta)=\sum_{\mathbf{x}} \Gamma_{\alpha \gamma}\left(x_{1}=n, \mathbf{x}, \beta\right)
$$

and

$$
\Gamma_{s \alpha \gamma}(n, \beta)=\Gamma_{\alpha \gamma}(n, \beta)-\sum_{m=0}^{5} \frac{\beta^{m}}{m !} \frac{d^{m}}{d \beta^{m}} \Gamma_{\alpha \gamma}(n, \beta=0) .
$$

Theorem III.2. For $\quad\left|\operatorname{Im} p_{1}\right|<-5 \ln c^{\prime} \beta, \quad\left|\Gamma_{s \alpha \gamma}(n, \beta)\right|<c|\beta|^{6}, \quad\left|\Gamma_{s \alpha \gamma}(n, \beta)\right| \leqq$ $c\left|c^{\prime} \beta\right|^{5 n}$ and $\Gamma_{s \alpha \gamma}(n, \beta)$ is analytic, $\tilde{\Gamma}\left(p_{1}, \beta\right)$ is jointly analytic and has the $\beta=0$ Taylor expansion

a) $\tilde{\Gamma}_{r r}\left(p_{1}, \beta\right)=2-4 g_{r_{1}} \beta+c_{2} \beta^{2}+\cdots c_{5} \beta^{5}+\left(-\frac{\beta^{4}}{8 r^{4}}-4\left(\alpha_{r}-\frac{g_{r_{1}}}{8 r^{4}}\right) \beta^{5}\right)$

$$
\cdot\left(e^{-i p_{1}}+e^{i p_{1}}\right)+\Gamma_{s r r}(n=0, \beta)+\sum_{n=1}^{\infty} \Gamma_{s r r}(n, \beta)\left(e^{-i p_{1} n}+e^{i p_{1} n}\right),
$$

b) $\widetilde{\Gamma}_{i i}\left(p_{1}, \beta\right)=2-4 g_{i_{1}} \beta+b_{2} \beta^{2}+\cdots b_{5} \beta^{5}+\left(-\frac{\beta^{4}}{8 r^{4}}-4\left(\alpha_{i}-\frac{g_{i_{1}}}{8 r^{4}}\right) \beta^{5}\right)$

$$
\cdot\left(e^{-i p_{1}}+e^{i p_{1}}\right)+\Gamma_{s i i}(n=0, \beta)+\sum_{n=1}^{\infty} \Gamma_{s i i}(n, \beta)\left(e^{-i p_{1} n}+e^{i p_{1} n}\right)
$$

c) $\tilde{\Gamma}_{r i}\left(p_{1}, \beta\right)=-4 a_{1} \beta+d_{2} \beta^{2}+\cdots d_{5} \beta^{5}-4\left(\alpha-\frac{a_{1}}{8 r^{4}}\right) \beta^{5}\left(e^{-i p_{1}}+e^{i p_{1}}\right)$

$$
+\Gamma_{s r i}(n=0, \beta)+\sum_{n=1}^{\infty} \Gamma_{s r i}(n, \beta)\left(e^{-i p_{1} n}+e^{i p_{1} n}\right),
$$

d) $\operatorname{det} \tilde{\Gamma}\left(p_{1}, \beta\right)=4\left[1-I_{1} \beta+I_{2} \beta^{2}+\cdots I_{5} \beta^{5}\right.$

$$
\left.+\left[-\frac{\beta^{4}}{8 r^{4}}+\left(4 \alpha_{i}-4 \gamma\right) \beta^{5}\right]\left(e^{-i p_{1}}+e^{i p_{1}}\right)\right]+0\left(\beta^{6}\right)
$$

where $I_{1}=2\left(g_{i_{1}}+g_{r_{1}}\right) ; I_{k}=I_{k}\left(g_{r_{k}}, \ldots, g_{r_{1}} ; g_{i_{k}}, \ldots, g_{i_{1}} ; a_{k-1}, \ldots, a_{1}\right), 2 \leqq k \leqq 5, b_{i}, c_{i}$, $d_{i}$ are group representation dependent constants.

Proof. The above is the Taylor expansion for $\tilde{\Gamma}\left(p_{1}, \beta\right)$ and the coefficients of the order $\beta^{m}$ terms, $0 \leqq m \leqq 5$, are determined from $\widetilde{\Gamma}\left(p_{1}, \beta\right) \widetilde{G}\left(p_{1}, \beta\right)=1$ using Thm. III.1. The analyticity properties and bounds follow from Lemma V.3. 
Introduce the auxiliary complex variable $w$ and $2 \times 2$ matrix function $H(w, \beta)$ such that $H_{\alpha \gamma}\left(w=2-\left(\beta^{4} / 8 r^{4}\right) e^{-i p_{1}}, \beta\right)=\widetilde{\Gamma}_{\alpha \gamma}\left(p_{1}, \beta\right)$ where

$$
\begin{aligned}
H_{r r}(w, \beta)= & w-12 g_{r_{1}} \beta+4 g_{r_{1}} w \beta+c_{r_{2}} \beta^{2}+\ldots c_{r_{5}} \beta^{5} \\
& -\frac{\beta^{8}}{64 r^{8}(2-w)}-\frac{g_{r_{1}} \beta^{9}}{16 r^{8}(2-w)}+K_{r r}(w, \beta), \\
H_{i i}(w, \beta)= & w-4\left(2 g_{r_{1}}+g_{i_{1}}\right) \beta+4 g_{r_{1}} w \beta+B_{i_{2}} \beta^{2}+\ldots B_{i_{5}} \beta^{5} \\
& -\frac{\beta^{8}}{64 r^{8}(2-w)}-\frac{g_{r_{1}} \beta^{9}}{16 r^{8}(2-w)}+K_{i i}(w, \beta), \\
H_{r i}(w, \beta)= & -12 a_{1} \beta+4 a_{1} w \beta+A_{2} \beta^{2}+\ldots A_{5} \beta^{5}-\frac{a_{1}}{16 r^{8}} \frac{\beta^{9}}{(2-w)}+K_{r i}(w, \beta),
\end{aligned}
$$

and we have set

$$
K_{\alpha \gamma}(w, \beta)=\Gamma_{s \alpha \gamma}(n=0, \beta)+\sum_{n=1}^{\infty} \Gamma_{s \alpha \gamma}(n, \beta)\left[\left(\frac{8 r^{4}(2-w)}{\beta^{4}}\right)^{n}+\left(\frac{\beta^{4}}{8 r^{4}(2-w)}\right)^{n}\right]
$$

The implicit equation we are trying to solve is now $F(w, \beta) \equiv \operatorname{det} H(w, \beta)=0$. Concerning $F(w, \beta)$ we have

Theorem III.3. For $|w|,|\beta|$ small

a) $F(w, \beta)$ is jointly analytic in $w, \beta$,

b) $F(0,0)=0, \partial F / \partial w(0,0)=0, \partial^{2} F / \partial w^{2}(0,0)=2$,

c) $F(w, \beta)=\left(A_{0}(\beta)+A_{1}(\beta) w+w^{2}\right) M(w, \beta)$, where $A_{0}(\beta), A_{1}(\beta)$ are analytic, $A_{0}(0)=A_{1}(0)=0 ; M(w, \beta)$ is jointly analytic and $M(w, \beta) \neq 0$.

Proof. a) Using the bounds of Theorem III.2 and the ratio test the infinite series converge absolutely. b) Similar to a). c) follows from a), b) and the Weierstrass preparation theorem (see Thm. 3.10 of [10]).

We now give the proof of Theorem A. From Theorem III.3c it is seen that the zeroes of $F(w, \beta)$ are given by the zeroes of $A_{0}(\beta)+A_{1}(\beta) w+w^{2}$. From [10] there are two possibilities:

1) There is only one zero given by $w(\beta)$, where $F(w(\beta), \beta)=0, w(0)=0$ and $w(\beta)$ is analytic.

2) There are two zeroes given by $w_{1}(\beta), w_{2}(\beta)$ with $w_{1}(0)=w_{2}(0)=0$, where $F\left(w_{1}(\beta), \beta\right)=F\left(w_{2}(\beta), \beta\right)=0$ and both $w_{1}(\beta)$ and $w_{2}(\beta)$ are analytic functions of $\beta$ or $\beta^{1 / 2}$.

As $F\left(w=2-\left(\beta^{4} / 8 r^{4}\right) e^{-i p_{1}}, \beta\right)=\operatorname{det} \tilde{\Gamma}\left(p_{1}, \beta\right)$, and using Lemma II.3 the zero or zeroes of det $\tilde{\Gamma}\left(p_{1}, \beta\right)$ corresponding to $p_{1}=\mathrm{im}>0$, are given by $m(\beta)=$ $-4 \ln \beta+r(\beta)=-4 \ln \beta+\ln 8 r^{4}+\ln (2-w(\beta)$, where $w(\beta)$ satisfies 1$)$ or 2$)$ above. In the case of two zeroes we are not asserting that both are given by $p_{1}$ pure imaginary and positive. However, there must be at least one zero as the mass spectrum is not empty since $\lim m_{\alpha \alpha} /-4 \ln \beta=1$ by Lemma V.1.

We remark that for an abelian group $G_{r i}=0$ and hence $\widetilde{G}_{r i}=\tilde{\Gamma}_{r i}=H_{r i}=0$. 
The implicit equations for the masses are $H_{r r}(w, \beta)=H_{i i}(w, \beta)=0$. There are unique analytic $w_{ \pm}(\beta)$ such that $H_{r r}\left(w_{+}(\beta), \beta\right)=H_{i i}\left(w_{-}(\beta), \beta\right)=0$ by the ordinary analytic implicit function theorem. However we don't know if mass splitting occurs, i.e. if $w_{ \pm}(\beta)$ are distinct.

\section{Mass Splitting and the Proof of Theorem B}

Here we obtain more detailed information on mass splitting and $\beta$ analyticity of the mass (rather than just $\beta^{1 / 2}$ analyticity) by an analysis of the terms quadratic in $w, \beta$ of the implicit mass equation $F(w, \beta)=\operatorname{det} H(w, \beta)=0$. We express various constants occurring in $F(w, \beta)$ in terms of $n_{D}$ and $n_{\bar{D}}$ in

\section{Lemma IV.1.}

a) $n_{D}=\int \chi^{2} \bar{\chi} d \mu=\int \bar{\chi}^{2} \chi d \mu$,

b) $n_{\bar{D}}=\int \chi^{3} d \mu=\int \bar{\chi}^{3} d \mu$,

c) $g_{r_{1}}=\frac{1}{8} \int(\chi+\bar{\chi})^{3} d \mu=\frac{1}{4}\left(n_{\bar{D}}+3 n_{D}\right)$,

d) $g_{i_{1}}=-\frac{1}{8} \int(\chi-\bar{\chi})^{2}(\chi+\bar{\chi}) d \mu=-\frac{1}{4}\left(n_{\bar{D}}-n_{D}\right)$,

e) $g_{r_{1}}-g_{i_{1}}=\frac{1}{2}\left(n_{D}+n_{\bar{D}}\right)$,

(f) $a_{1}=-\frac{i}{8} \int(\chi-\bar{\chi})(\chi+\bar{\chi})^{2} d \mu=-\frac{i}{8}\left(n_{\bar{D}}+n_{D}-n_{D}-n_{\bar{D}}\right)=0$.

We have

\section{Theorem IV.1}

$$
F(w, \beta)=\operatorname{det} H(w, \beta)=\left(w-\alpha_{+} \beta\right)\left(w-\alpha_{-} \beta\right)-T(w, \beta),
$$

where

$$
\alpha_{ \pm}=(2 \pm 1) n_{\bar{D}}+(8 \pm 1) n_{D}, \alpha_{+}-\alpha_{-}=2\left(n_{\bar{D}}+n_{D}\right)
$$

and

$$
T(w, \beta)=\sum_{3 \leqq \imath+j} a_{i j} \beta^{i} w^{j}
$$

is jointly analytic in $w, \beta$.

Proof. As $\quad \operatorname{det} H(w, \beta)=w^{2}-4\left(5 g_{r_{1}}+g_{i_{1}}\right) w \beta+\left[48 g_{r_{1}}\left(2 g_{r_{1}}+g_{i_{1}}\right)-144 a_{1}^{2}\right] \beta^{2}-$ $T(w, \beta)$ the result follows using Lemma IV.1.

Theorem IV.2. If $n_{D}+n_{\bar{D}}>0$ there exist two distinct analytic functions $w_{ \pm}(\beta)=\alpha_{ \pm} \beta$ $+O\left(\beta^{2}\right)$ such that $\operatorname{det} H\left(w_{ \pm}(\beta), \beta\right)=0$.

Proof. The roots of the quadratic form in Thm. IV.1 are distinct by assumption. For simplicity we write (see p. 97 of [12])

$$
\operatorname{det} H(w, \beta)=w^{2}+a_{11} \beta w+a_{20} \beta^{2}-T(w, \beta)=0 .
$$


Then $a_{11}^{2}-4 a_{20} \neq 0$ and $2 \alpha_{+}+a_{11}=\left(a_{11}^{2}-4 a_{20}\right)^{1 / 2}=\alpha_{+}-\alpha_{-} \neq 0$. Make the transformation $w_{+}=\beta\left(\alpha_{+}+v\right)$. Then, with $w=w_{+},(*)$ becomes

$$
\beta^{2}\left(\alpha_{+}+v\right)^{2}+a_{11} \beta^{2}\left(\alpha_{+}+v\right)+a_{20} \beta^{2}=\sum_{3 \leqq j+k} a_{j k} \beta^{j} \beta^{k}\left(\alpha_{+}+v\right)^{k},
$$

and cancelling $\beta^{2}$ gives

$$
\left(\alpha_{+}^{2}+2 \alpha_{+} v+v^{2}\right)+a_{11}\left(\alpha_{+}+v\right)+a_{20}=\sum_{3 \leqq j+k} a_{j k} \beta^{j+k-2}\left(\alpha_{+}+v\right)^{k}
$$

or

$$
v\left(2 \alpha_{+}+a_{11}+v\right)=v\left(\alpha_{+}-\alpha_{-}+v\right) \equiv \beta L(\beta, v),
$$

with $L(\beta, v)$ jointly analytic. Let $R(\beta, v)=v\left(\alpha_{+}-\alpha_{-}+v\right)-\beta L(\beta, v)$. Then $R(0,0)=0$, $\partial R / \partial v(0,0)=\alpha_{+}-\alpha_{-} \neq 0$, so by the analytic implicit function theorem there exists a unique analytic $v(\beta), v(0)=0$, such that det $H\left(w_{+}(v(\beta)), \beta\right)=0$. Similarly we make the transformation $w_{-}=\beta\left(\alpha_{-}+u\right)$ and get a unique analytic $u(\beta)$.

We now give the proof of Theorem $B$. Note that

$$
H_{r r}(w, \beta)=w-\alpha_{+} \beta+\sum_{2 \leqq i+j} b_{i j} \beta^{i} w^{j}, \quad H_{i i}(w, \beta)=w-\alpha_{-} \beta+\sum_{2 \leqq i+j} c_{i j} \beta^{i} w^{j},
$$

and $H_{r i}(w, \beta)=\sum_{2 \leqq i+j} d_{i j} \beta^{i} w^{j}$, so that from Lemma IV.1 and Theorem IV.2

$$
\begin{array}{ll}
H_{r r}\left(w_{+}(\beta), \beta\right)=0\left(\beta^{2}\right), & H_{i i}\left(w_{+}(\beta), \beta\right)=\left(\alpha_{+}-\alpha_{-}\right) \beta+0\left(\beta^{2}\right), \\
H_{i i}\left(w_{-}(\beta), \beta\right)=0\left(\beta^{2}\right), & H_{r r}\left(w_{-}(\beta), \beta\right)=\left(\alpha_{-}-\alpha_{+}\right) \beta+0\left(\beta^{2}\right) .
\end{array}
$$

Thus by Lemma II. 3, as $H_{i i}\left(w_{+}(\beta), \beta\right) \neq 0$ and $H_{r r}\left(w_{-}(\beta), \beta\right) \neq 0$ for small $\beta>0$, we have two distinct masses $m_{ \pm}(\beta)$ given by $\beta^{4} e^{m_{ \pm}(\beta)} / 8 r^{4}=2-w_{ \pm}(\beta)$ or

$$
m_{ \pm}(\beta)=\ln \left[\frac{8 r^{4}}{\beta^{4}}\left(2-w_{ \pm}(\beta)\right)\right] \text {. }
$$

For $\mathrm{SU}(3)$, as $3 \times 3=6+3^{*}, n_{\bar{D}}=1$.

The determination of the $\left\{c_{n}\right\}$ follows along the lines of $[2,3]$

\section{Decay Properties of $\boldsymbol{G}(\boldsymbol{x}, \beta)$ and $\Gamma(x, \beta)$}

In this section we establish $\beta$-analyticity and decay properties of $G(x, \beta)$ and $\Gamma(x, \beta)$, which follow from the corresponding properties of the finite lattice $G_{\Lambda}(0 ; x, \beta)$ and $\Gamma_{\Lambda}(0 ; x, \beta)$. We also determine the coefficients of the $\beta=0$ expansion of $G(x, \beta)$ up to and including order $\beta^{5}$. We use the complex coupling parameters and notation of [1]. In what follows we repeatedly use the Peter-Weyl group orthogonality relations $(\mathrm{P}-\mathrm{W})$ for representations for complex character, i.e.

$$
\begin{gathered}
\int \bar{D}_{i j}(g) D_{k l}(g) d \mu=\frac{1}{r} \delta_{i k} \delta_{j l}, \quad \int D_{i j}(g) D_{k l}(g) d \mu=0, \\
\int\left|\chi_{r}(g)\right|^{2} d \mu=\int\left|\chi_{i}(g)\right|^{2} d \mu=\frac{1}{2}, \quad \int \chi_{r}(g) \chi_{i}(g) d \mu=0,
\end{gathered}
$$

where $D_{i j}(g)$ denote the matrix elements of the unitary representative of the group element $g$. Note also that for a complex character $\chi(g)=\bar{\chi}\left(g^{-1}\right)$, so that $\chi_{r}\left(g_{p}\right)=$ 
$\chi_{r}\left(g_{p}^{-1}\right)$ but $\chi_{i}\left(g_{p}\right)=-\chi_{i}\left(g_{p}^{-1}\right)$ and $g_{p}^{-1}$ is the plaquette oriented border product in the opposite sense to that of $g_{p}$. We give only the proofs that don't require major modifications of analogous ones in $[1,2]$.

\section{Lemma V.1}

a) $G_{\alpha \gamma}(x, \beta)$ is analytic in $\beta$ and $\left|G_{\alpha \gamma}(x, \beta)\right| \leqq c_{1}|c \beta|^{|| x_{1}|+| x \mid}$,

b) For $\beta>0$ and small $G_{\alpha \alpha}\left(x_{1}, \mathbf{x}=0, \beta\right)>c_{3}\left|c_{4} \beta\right|^{4\left|x_{1}\right|}$,

c) $m_{\alpha \alpha}=\lim _{x_{1} \rightarrow \infty}-\frac{1}{x_{1}} \ln G_{\alpha \alpha}\left(x=\left(x_{1}, 0\right), \beta\right)$ exists and $\lim _{\beta \downarrow 0} \frac{m_{\alpha \alpha}}{-4 \ln \beta}=1$.

Proof. Similar to [1] except that in b) the integral over the sides of an elementary cube is performed as in the proof of Lemma V.2b.

Lemma V.2. Set $G^{m}(x, 0) \equiv(1 / m !)\left(d^{m} / d \beta^{m}\right) G(x, \beta=0)$. Then for

a) $x=0$ :

$$
\begin{array}{ll}
G_{r r}^{0}=\frac{1}{2}, & G_{r r}^{1}=\int \chi_{r}^{3} d \mu, \\
G_{i i}^{0}=\frac{1}{2}, & G_{i i}^{1}=\int \chi_{1}^{2} \chi_{r} d \mu, \\
G_{r i}^{0}=0, & G_{r i}^{1}=\int \chi_{i} \chi_{r}^{2} d \mu,
\end{array}
$$

b) $x=(1, \mathbf{0})$ :

$$
G_{r r}^{4}=\frac{1}{32 r^{4}}=G_{i i}^{4}, \quad G_{r i}^{4}=0,
$$

c) $x=(1,0)$ :

$$
G_{r r}^{5}=\frac{1}{4 r^{4}} \int \chi_{r}^{3} d \mu, \quad G_{i i}^{5}=\frac{1}{8 r^{4}}\left(\int\left[\chi_{r}^{3}+\chi_{i}^{2} \chi_{r}\right] d \mu \quad G_{r i}^{5}=\frac{1}{4 r^{4}} \int \chi_{r}^{2} \chi_{i} d \mu .\right.
$$

Proof. The $G_{\alpha \gamma}^{m}$ results follow from the $\beta=0$ expansion of $G_{\Lambda \alpha \gamma}(0 ; x, \beta)$. Note that $\left\langle\chi_{\alpha}\left(g_{p}\right)\right\rangle_{A}=\left(1-\delta_{\alpha i}\right) \beta / 2+0\left(\beta^{2}\right)$ and that the denominator of \langle\rangle$_{A}$ is $1+O\left(\beta^{2}\right)$. a) The $G_{\alpha \gamma}^{0}$ and $G_{\alpha \gamma}^{1}$ values follow from expanding the numerator of $\left\langle\chi_{\alpha}\left(g_{p_{0}}\right) \chi_{\gamma}\left(g_{p_{0}}\right)\right\rangle_{\Lambda}$ and using P-W.

b) As in [1] write \langle\rangle$_{A}$ in terms of duplicate variables and expand the numerator to get

$$
\begin{aligned}
m ! G_{\Lambda \alpha \gamma}^{m}(x, 0)= & \frac{1}{2} \sum_{p_{1} \ldots p_{m}} \int\left(\chi_{\alpha}\left(g_{p_{0}}\right)-\chi_{\alpha}\left(g_{p_{0}}^{\prime}\right)\right)\left(\chi_{\gamma}\left(g_{p_{x}}\right)-\chi_{\gamma}\left(g_{p_{x}}^{\prime}\right)\right) \\
& \cdot \prod_{i=1}^{m}\left(\chi_{r}\left(g_{p_{i}}\right)+\chi_{r}\left(g_{p_{i}}^{\prime}\right)\right) d g_{\Lambda} d g_{\Lambda}^{\prime}
\end{aligned}
$$

for $m=4$ and $m=5$. For $m=4$ the only plaquettes that contribute are the ones forming the 4 sides of a cube containing $P_{0}$ and $P_{x}$. We denote them by $P^{a}, 1 \leqq a \leqq 4$. 
All the cross terms vanish so that, counting the 4 ! permutations of the $P^{a}$, we have

$$
G_{\Lambda \alpha \gamma}^{4}(x, 0)=\int \chi_{\alpha}\left(g_{p_{0}}\right) \chi_{\gamma}\left(g_{p_{x}}\right) \prod_{a=1}^{4} \chi_{r}\left(g_{p} a\right) d g_{\Lambda} .
$$

We integrate over the group variables of the four bonds parallel to the 1-direction in $\bigcup_{a} P^{a}$, successively in the positive sense, to get, by $\mathrm{P}-\mathrm{W}$,

$$
\frac{1}{8 r^{4}} \operatorname{Re}\left(\chi\left(g_{p_{0}}\right) \chi\left(g_{p_{x}}^{-1}\right)\right)=\frac{1}{8 r^{4}}\left[\chi_{r}\left(g_{p_{0}}\right) \chi_{r}\left(g_{p_{x}}^{-1}\right)-\chi_{i}\left(g_{p_{0}}\right) \chi_{i}\left(g_{p_{x}}^{-1}\right)\right] .
$$

Substituting in $G_{\alpha \gamma}^{4}(x, 0)$ gives

$$
G_{\Lambda \alpha \gamma}^{4}(x, 0)=\frac{1}{8 r^{4}} \int \chi_{\alpha}\left(g_{p_{0}}\right) \chi_{\gamma}\left(g_{p_{x}}\right)\left[\chi_{r}\left(g_{p_{0}}\right) \chi_{r}\left(g_{p_{x}}^{-1}\right)-\chi_{i}\left(g_{p_{0}}\right) \chi_{i}\left(g_{p_{x}}^{-1}\right)\right] d g_{\Lambda} .
$$

By using $\chi_{r}\left(g_{p_{x}}^{-1}\right)=\chi_{r}\left(g_{p_{x}}\right), \chi_{i}\left(g_{p_{x}}^{-1}\right)=-\chi_{i}\left(g_{p_{x}}\right)$ and $\mathrm{P}-\mathrm{W}$ the result follows.

c) For $m=5$ the only plaquettes that contribute are the four $P^{a}$ s and a fifth plaquette, call it $P^{5}$, which can be a $P^{a}$ or $P_{0}$ or $P_{x}$. Again no cross terms contribute. If $P^{5}$ is a $P_{0}$ or $P_{x}$ we integrate as before to obtain the contribution (for 1 term in the $\operatorname{sum} \sum_{p_{1} \ldots p_{5}}$ )

$$
\begin{aligned}
& \frac{1}{5 !} \frac{1}{16 r^{4}} \int \chi_{r}^{3} d \mu \quad \text { for } \alpha \gamma=r r, \\
& \frac{1}{5 !} \frac{1}{16 r^{4}} \int \chi_{i}^{2} \chi_{r} d \mu \quad \text { for } \alpha \gamma=i i, \\
& \frac{1}{5 !} \frac{1}{16 r^{4}} \int \chi_{r}^{2} \chi_{i} d \mu \quad \text { for } \alpha \gamma=r i .
\end{aligned}
$$

Taking into account permutations there are $2 \cdot 5$ ! terms.

If the fifth plaquette is a $P^{a}$ then we integrate over the 4 bonds around the sides of the cube not containing $P^{a}$ to get (for 1 term)

$$
\begin{aligned}
& \frac{1}{5 !} \frac{1}{16 r^{4}} \int \chi_{r}^{3} d \mu, \quad \alpha \gamma=r r \text { or } i i, \\
& \frac{1}{5 !} \frac{1}{16 r^{4}} \int \chi_{r}^{2} \chi_{i} d \mu, \quad \alpha \gamma=r i .
\end{aligned}
$$

There are 2.5! such terms and summing these contributions with the contributions above gives the result.

Lemma V.3. $\Gamma_{\alpha \gamma}(x, \beta)$ is analytic in $\beta$ and $\left|\Gamma_{\alpha \gamma}(x, \beta)\right| \leqq c_{2}\left|c^{\prime} \beta\right|^{5\left|x_{1}\right|+|x|}, x \neq( \pm 1,0$ or $x=( \pm 1,0), \alpha \neq \gamma$; for $x=( \pm 1,0)$ and $\alpha=\gamma$ replace the 5 by 4 .

Proof. The existence and $\beta$-analyticity of $\Gamma(x, \beta)$ follows from that of $\Gamma_{\Lambda}(x ; y, \beta)$ which follows as in Lemma 4.9 of [1], remembering that here $\Gamma_{A}$ stands for the operator with matrix elements $\Gamma_{\Lambda \alpha \gamma}(x ; y, \beta)$. The proof of the bound on $\Gamma_{\alpha \gamma}(x, \beta)$ follows that of Theorems 4.11 and 4.12 of [1] except that we append indices $\alpha, \gamma$ 
to $G$ and $\Gamma$ and products include $2 \times 2$ matrix multiplication. In more detail, for $x_{1} \leqq p<y_{1}, u_{1} \leqq p<v_{1}$ and $0 \leqq m \leqq 3$,

$$
\left.\frac{\partial^{m}}{\partial w_{p}^{m}} G_{\Lambda \alpha \gamma}(u ; v)\right|_{w_{p}=0}=\left.\frac{\partial^{m}}{\partial w_{p}^{m}} \Gamma_{\Lambda \alpha \gamma}(u ; v)\right|_{w_{p}=0}=0
$$

and

$$
\begin{aligned}
& \left.\frac{\partial^{4}}{\partial w_{p}^{4}} G_{\Lambda \alpha \gamma}(u ; v)\right|_{w_{p}=0}=\frac{4 !}{\left.2 Z_{\Lambda}^{2}\right|_{w_{p}=0}} \sum_{a \in C_{p, \Lambda}} \iint\left(\chi_{\alpha}\left(g_{u}\right)-\chi_{\alpha}\left(g_{p_{u}}^{\prime}\right)\right) \\
& \cdot\left(\chi_{\gamma}\left(g_{p_{v}}\right)-\chi_{\gamma}\left(g_{p_{v}}^{\prime}\right)\right) \cdot\left[\prod_{b=1}^{4} \chi_{r}\left(g_{p_{b}^{a}}\right)+\prod_{b=1}^{4} \chi\left(g_{p_{b}^{a}}^{\prime}\right)\right] \\
& \cdot \exp \left[z \sum_{p \in p_{\perp}}\left(\chi_{r}\left(g_{p}\right)+\chi_{r}\left(g_{p}^{\prime}\right)\right)+\sum_{\substack{q=-N \\
q \neq p}}^{N-1} w_{q} \sum_{p \in P_{\|}, \Lambda}\left(\chi_{r}\left(g_{p}\right)+\chi_{r}\left(g_{p}^{\prime}\right)\right)\right] d g_{\Lambda} d g_{\Lambda}^{\prime} .
\end{aligned}
$$

Here $C_{p, \Lambda}$ denotes the collection of elementary cubes between the planes $x_{1}=p$ and $x_{1}=p+1$ and $p_{b}^{a}, 1 \leqq b \leqq 4$, are the plaquettes in the four faces of the cube a parallel to the $x_{1}$ direction.

Integrating over the group variables parallel to the one-direction of $\left\{P_{b}^{a}\right\}$ as in the proof of Lemma V.2b gives

$$
\int \prod_{b=1}^{4} \chi_{r}\left(g_{p_{b}^{a}}\right) d g_{a}=\frac{1}{8 r^{4}}\left[\chi_{r}\left(g_{p_{<}^{a}}\right) \chi_{r}\left(g_{p_{>}^{a}}^{-1}\right)-\chi_{i}\left(g_{p_{<}^{a}}\right) \chi_{i}\left(g_{p_{>}^{a}}^{-1}\right)\right] .
$$

Here $P_{<}^{a}\left(P_{>}^{a}\right)$ is the plaquette in the face of the cube a (between the planes $x_{1}=p$ and $\left.x_{1}=p+1\right)$ perpendicular to the 1-direction in the $x_{1}=p\left(x_{1}=p+1\right)$ plane; $g_{p_{<}^{a}}\left(g_{p_{>}^{a}}^{-1}\right)$ is the positively (negatively) oriented group element product around the border of the plaquette $P_{<}^{a}\left(P_{>}^{a}\right)$. Substituting in the above, remembering that $G_{\Lambda \alpha \gamma}$ is defined with positively oriented plaquettes and that $\chi_{i}\left(g_{P a}^{-1}\right)=-\chi_{i}\left(g_{P_{>}^{a}}\right)$, gives

$$
\left.\frac{\partial^{4}}{\partial w_{q}^{4}} G_{\Lambda \alpha \gamma}(u ; v)\right|_{w_{p}=0}=\left.\left.\frac{4 !}{8 r^{4}} \sum_{t_{1}=\rho} G_{\Lambda \alpha \rho}(u, t)\right|_{w_{p}=0} G_{\Lambda \rho \gamma}\left(t+\hat{e}_{1}, v\right)\right|_{w_{p}=0}
$$

Thus

$$
\begin{aligned}
\left.\frac{\partial^{4}}{\partial w_{p}^{4}} \Gamma_{\Lambda \alpha \gamma}(x ; y)\right|_{w_{p}=0} & =\sum_{u, v \in \Lambda}\left[\left.\left.\left.\Gamma_{\Lambda}(x ; u)\right|_{w_{p}=0} \frac{\partial^{4} G}{\partial w_{p}^{4}}(u, v)\right|_{w_{p}=0} \Gamma_{\Lambda}(v ; y)\right|_{w_{p}=0}\right]_{\alpha \gamma} \\
& =\frac{4 !}{8 r^{4}} \sum_{t_{1}=p} \delta_{x, t} \delta_{t+e_{1}, y} \delta_{\alpha \gamma}=\frac{4 !}{8 r^{4}} \delta_{x+e_{1}, y} \delta_{x_{1}, p} \delta_{\alpha \gamma}
\end{aligned}
$$

from which the bound follows as in Theorem 4.12 of [1].

\section{Concluding Remarks}

In the context of Theorem $\mathrm{A}$ the question arises as to the $\beta$ analyticity of the mass or masses. In the case of the $\beta$-analytic $2 \times 2$ matrix eigenvalue problem Rellich's theorem says that if the matrix is self-adjoint for $\beta$ real then the eigenvalues are 
analytic in $\beta$ (see [11]). Does an analogue of Rellich's theorem hold here? Is there mass splitting in the abelian case? Also our results should generalize to dispersion curves.

There is also the question of what happens in $3+1$ dimensions, i.e. how is the glueball mass spectrum affected by taking into account $\chi$ and $\bar{\chi}$ ? Last but not least what happens for $\beta$ large?

\section{References}

1. Schor, R.: Nucl. Phys. B. Vol. 222, 71-82. July 18, 1983

2. O’Carroll, M., Braga, G.: J. Math. Phys. 25, 2741-2743 (1984)

3. O'Carroll, M.: Analyticity properties and a convergent expansion for the inverse correlation length of the high temperature $d$-dimensional Ising model J. Stat. Phys. Vol. 34, 597-608 (1984); Phys. Letts. B143, 188-192 (1984)

4. Munster, G.: Nucl. Phys. B190, 439-453 (1981)

5. Berg, B. et al.: Nucl. Phys. B221, 109-140 (1983)

6. Osterwalder, K., Seiler, E.: Ann. Phys. 110, 440 (1978)

7. Seiler, E.: Gauge theories as a problem of constructive quantum field theory and statistical mechanics. In: Lecture Notes in Physics, Vol. 159. New York: Springer, 1982

8. Schor, R.: Commun. Math. Phys. 59, 213 (1978)

9. Paes-Leme, P.J.: Ann. Phys. 115, 367-387(1978)

10. Markushevich, A. I.: Theory of functions of a complex variable, Vol. II. Engle-wood Cliffs, New Jersey: Prentice-Hall, 1965

11. Reed, M., Simon, S.: Methods of modern mathematical physics, Vol. IV. New York: Academic Press, 1978

12. Hille, E.: Analytic functions, Vol. II. Ginn and Company 1962

Communicated by A. Jaffe

Received May 24, 1984; in revised form July 19, 1984

Note added in proof. Theorem A can be strengthened to $r(\beta)$ is analytic at $\beta=0$. By remark 3 after Theorem B it is easy to show that for $\vec{p}=0 \widetilde{G}_{r i}\left(p_{1}\right)=0$ so that $\operatorname{det} \tilde{\Gamma}\left(p_{1}\right)$ factors, i.e. $\operatorname{det} \tilde{\Gamma}\left(p_{1}\right)=$ $\widetilde{\Gamma}_{r r}\left(p_{1}\right) \tilde{\Gamma}_{i i}\left(p_{1}\right)$. The mass spectrum is contained in $\widetilde{\Gamma}_{r r}\left(p_{1}, \beta\right)=0$ and $\widetilde{\Gamma}_{i i}\left(p_{1}, \beta\right)=0, \operatorname{Im} p_{1}>0$, which can be solved by the methods of $[2,3]$. However, there are multi-component classical lattice spin systems where $\operatorname{det} \widetilde{\Gamma}\left(p_{1}\right)$ does not factor, i.e. an anisotropic rotor in a uniform magnetic field and the mass spectrum is contained in $\operatorname{det} \tilde{\Gamma}\left(p_{1}\right)=0, \operatorname{Im} p_{1}>0$ (see O'Carroll, M. J. Stat. Phys. Vol. 37, 439-449 (1984)). 